\title{
EXt-SYMMETRY OVER QUANTUM COMPLETE INTERSECTIONS
}

\author{
PETTER ANDREAS BERGH
}

\begin{abstract}
We show that symmetry in the vanishing of cohomology holds for graded modules over quantum complete intersections. Moreover, symmetry holds for all modules if the algebra is symmetric.
\end{abstract}

\section{INTRODUCTION}

For which algebras do symmetry in the vanishing of cohomology hold? That is, given an algebra $\Lambda$, does the implication

$$
\operatorname{Ext}_{\Lambda}^{i}(M, N)=0 \text { for } i \gg 0 \Rightarrow \operatorname{Ext}_{\Lambda}^{i}(N, M)=0 \text { for } i \gg 0
$$

hold for finitely generated modules $M$ and $N$ ? As shown in AvB, this implication holds for finitely generated modules over commutative local complete intersections. The proof involves the theory of certain support varieties attached to each pair of finitely generated modules over such a ring $A$. Namely, denote by $c$ the codimension of $A$ and by $K$ the algebraic closure of its residue field. A cone $\mathrm{V}(M, N)$ in $K^{c}$ is associated to every pair $(M, N)$ of finitely generated $A$-modules, with the following properties:

$$
\begin{aligned}
\mathrm{V}(M, N)=\{0\} & \Leftrightarrow \quad \operatorname{Ext}_{A}^{i}(M, N)=0 \text { for } i \gg 0 \\
\mathrm{~V}(M, N) & =\mathrm{V}(M, M) \cap \mathrm{V}(N, N) .
\end{aligned}
$$

The symmetry in the vanishing of cohomology follows immediately from these properties. As shown in [Mor, Corollary 4.8], group algebras of finite groups provide another class of examples where Ext-symmetry holds.

We show in this paper that Ext-symmetry holds for all graded modules over quantum complete intersections, provided all the defining commutators are roots of unity. We also show that, if such an algebra is symmetric, that is, if it is isomorphic as a bimodule to its own dual, then symmetry holds for all modules.

\section{Quantum Complete intersections}

Quantum complete intersections are noncommutative analogues of truncated polynomial rings. Included in this class of algebras are exterior algebras and finite dimensional complete intersections of the form $k\left[X_{1}, \ldots, X_{c}\right] /\left(X_{1}^{a_{1}}, \ldots, X_{c}^{a_{c}}\right)$. Fix a field $k$, let $c \geq 1$ be an integer, and let $\mathbf{q}=\left(q_{i j}\right)$ be a $c \times c$ commutation matrix with entries in $k$. That is, the diagonal entries $q_{i i}$ are all 1 , and $q_{i j} q_{j i}=1$ for all $i, j$. Furthermore, let $\mathbf{a}_{c}=\left(a_{1}, \ldots, a_{c}\right)$ be an ordered sequence of $c$ integers with $a_{i} \geq 2$. The quantum complete intersection $A_{\mathbf{q}}^{\mathbf{a}_{c}}$ determined by these data is the algebra

$$
A_{\mathbf{q}}^{\mathbf{a}_{c}} \stackrel{\text { def }}{=} k\left\langle X_{1}, \ldots, X_{c}\right\rangle /\left(X_{i}^{a_{i}}, X_{i} X_{j}-q_{i j} X_{j} X_{i}\right) \text {. }
$$

2000 Mathematics Subject Classification. 16E30, 16S80, 16U80, 81R50.

Key words and phrases. Quantum complete intersections, vanishing of cohomology, symmetry. The author was supported by NFR Storforsk grant no. 167130 . 
This is a finite dimensional selfinjective algebra of dimension $\prod_{i=1}^{c} a_{i}$. The image of $X_{i}$ in this quotient will be denoted by $x_{i}$. We shall consider $A_{\mathbf{q}}^{\mathbf{a}_{c}}$ as a $\mathbb{Z}^{c}$-graded algebra, in which the degree of the generator $x_{i}$ is the $i$ th unit vector $(0, \ldots, 1, \ldots 0)$. The category of finitely generated left $A_{\mathbf{q}}^{\mathbf{a}_{c}}$-modules (respectively, graded modules) is denoted by $\bmod A_{\mathbf{q}}^{\mathbf{a}_{c}}$ (respectively, $\operatorname{grmod} A_{\mathbf{q}}^{\mathbf{a}_{c}}$ ); all modules are assumed to be finitely generated.

A quantum complete intersection is built from truncated polynomial rings using certain tensor products. We recall here the basics, details can be found in [BeO]. Let $A$ and $B$ be abelian groups, and let $\Lambda$ and $\Gamma$ be an $A$-graded and a $B$-graded $k$-algebra, respectively. Furthermore, let $t: A \otimes_{\mathbb{Z}} B \rightarrow k \backslash\{0\}$ be a homomorphism of groups, where $k \backslash\{0\}$ is multiplicative. If $a$ and $b$ are elements of $A$ and $B$, respectively, then we write $t(a \mid b)$ instead of $t(a \otimes b)$. Moreover, given homogeneous elements $\lambda \in \Lambda$ and $\gamma \in \Gamma$ of degrees $d$ and $d^{\prime}$, respectively, we write $t(\lambda \mid \gamma)$ instead of $t\left(d \mid d^{\prime}\right)$. With these data, we can now define a new algebra $\Lambda \otimes_{k}^{t} \Gamma$, the twisted tensor product of $\Lambda$ and $\Gamma$ with respect to the homomorphism $t$. The underlying $k$-vector space of $\Lambda \otimes_{k}^{t} \Gamma$ is $\Lambda \otimes_{k} \Gamma$, and multiplication is given by

$$
\left(\lambda_{1} \otimes \gamma_{1}\right) \cdot\left(\lambda_{2} \otimes \gamma_{2}\right) \stackrel{\text { def }}{=} t\left(\lambda_{2} \mid \gamma_{1}\right) \lambda_{1} \lambda_{2} \otimes \gamma_{1} \gamma_{2}
$$

for homogeneous elements $\lambda_{1}, \lambda_{2} \in \Lambda$ and $\gamma_{1}, \gamma_{2} \in \Gamma$. This algebra is $A \oplus B$-graded; if $a \in A$ and $b \in B$, then $\left(\Lambda \otimes_{k}^{t} \Gamma\right)_{(a, b)}=\Lambda_{a} \otimes_{k} \Gamma_{b}$. Given a graded $\Lambda$-module $M$ and a graded $\Gamma$-module $N$, their tensor product $M \otimes_{k} N$ becomes a graded $\Lambda \otimes_{k}^{t} \Gamma$-module by defining

$$
(\lambda \otimes \gamma) \cdot(m \otimes n) \stackrel{\text { def }}{=} t(m \mid \gamma) \lambda m \otimes \gamma n
$$

for homogeneous elements $\lambda \in \Lambda, \gamma \in \Gamma, m \in M, n \in N$. This module is denoted $M \otimes_{k}^{t} N$. As above, this module is $A \oplus B$-graded; if $a \in A$ and $b \in B$, then $\left(M \otimes_{k}^{t} N\right)_{(a, b)}=M_{a} \otimes_{k} N_{b}$.

The purpose of this paper is to study symmetry in the vanishing of cohomology over quantum complete intersections. We therefore end this section with the following two results, the first of which shows that quantum complete intersections are made up of twisted tensor products.

Lemma 2.1. Let $A_{\mathbf{q}}^{\mathbf{a}_{c}}$ be a quantum complete intersection with $c \geq 2$, let $I$ be a proper nonempty subset of $\{1, \ldots, c\}$ of order $c_{1}$, and let $c_{2}=c-c_{1}$. Furthermore, let $A_{\mathbf{q}_{1}}^{\mathbf{a}_{c_{1}}}$ and $A_{\mathbf{q}_{2}}^{\mathbf{a}_{c_{2}}}$ be the quantum complete intersections generated by $\left\{x_{i}\right\}_{i \in I}$ and $\left\{x_{i}\right\}_{i \in\{1, \ldots, c\} \backslash I}$, respectively. Then there is an isomorphism

$$
A_{\mathbf{q}}^{\mathbf{a}_{c}} \simeq A_{\mathbf{q}_{1}}^{\mathbf{a}_{c_{1}}} \otimes_{k}^{t} A_{\mathbf{q}_{2}}^{\mathbf{a}_{c_{2}}}
$$

for some homomorphism $\mathbb{Z}^{c_{1}} \otimes_{\mathbb{Z}} \mathbb{Z}^{c_{2}} \rightarrow k \backslash\{0\}$.

Proof. By re-indexing the generators, we may assume that $I=\left\{1, \ldots, c_{1}\right\}$. Thus $A_{\mathbf{q}_{1}}^{\mathbf{a}_{c_{1}}}$ is the subalgebra of $A_{\mathbf{q}}^{\mathbf{a}_{c}}$ generated by $x_{1}, \ldots, x_{c_{1}}$, whereas $A_{\mathbf{q}_{2}}^{\mathbf{a}_{c_{2}}}$ is the subalgebra generated by the $c_{2}$ elements $x_{c_{1}+1}, \ldots, x_{c}$.

Consider the map $\mathbb{Z}^{c_{1}} \times \mathbb{Z}^{c_{2}} \stackrel{t^{\prime}}{\rightarrow} k \backslash\{0\}$ defined by

$$
\left(\left(d_{1}, \ldots, d_{c_{1}}\right),\left(d_{c_{1}+1}, \ldots, d_{c}\right)\right) \mapsto \prod_{j=c_{1}+1}^{c} \prod_{i=1}^{j-1} q_{j i}^{d_{i} d_{j}} .
$$

Given sequences $\mathbf{d}_{1}, \mathbf{d}_{1}^{\prime} \in \mathbb{Z}^{c_{1}}$ and $\mathbf{d}_{2}, \mathbf{d}_{2}^{\prime} \in \mathbb{Z}^{c_{2}}$, the equalities

$$
\begin{aligned}
t^{\prime}\left(\mathbf{d}_{1}+\mathbf{d}_{1}^{\prime}, \mathbf{d}_{2}\right) & =t^{\prime}\left(\mathbf{d}_{1}, \mathbf{d}_{2}\right) t^{\prime}\left(\mathbf{d}_{1}^{\prime}, \mathbf{d}_{2}\right) \\
t^{\prime}\left(\mathbf{d}_{1}, \mathbf{d}_{2}+\mathbf{d}_{2}^{\prime}\right) & =t^{\prime}\left(\mathbf{d}_{1}, \mathbf{d}_{2}\right) t^{\prime}\left(\mathbf{d}_{1}, \mathbf{d}_{2}^{\prime}\right)
\end{aligned}
$$

hold, hence $t^{\prime}$ induces a homomorphism $\mathbb{Z}^{c_{1}} \otimes_{\mathbb{Z}} \mathbb{Z}^{c_{2}} \stackrel{t}{\rightarrow} k \backslash\{0\}$ of abelian groups. Now let $i$ and $j$ be elements of $I$, and consider the elements $x_{i} \otimes 1$ and $x_{j} \otimes 1$ in 
the twisted tensor product $A_{\mathbf{q}_{1}}^{\mathbf{a}_{c_{1}}} \otimes_{k}^{t} A_{\mathbf{q}_{2}}^{\mathbf{a}_{c_{2}}}$. The degrees of $x_{i} \in A_{\mathbf{q}_{1}}^{\mathbf{a}_{c_{1}}}$ and $x_{j} \in A_{\mathbf{q}_{1}}^{\mathbf{a}_{c_{1}}}$ are the unit vectors $e_{i}$ and $e_{j}$ in $\mathbb{Z}^{c_{1}}$, respectively, whereas the degree of $1 \in A_{\mathbf{q}_{2}}^{\mathbf{a}_{c_{2}}}$ is the zero vector in $\mathbb{Z}^{c_{2}}$. Therefore

$$
\begin{aligned}
\left(x_{i} \otimes 1\right)\left(x_{j} \otimes 1\right) & =t\left(e_{j} \mid 0\right) x_{i} x_{j} \otimes 1 \\
& =x_{i} x_{j} \otimes 1 \\
& =q_{i j} x_{j} x_{i} \otimes 1 \\
& =q_{i j} t\left(e_{i} \mid 0\right) x_{j} x_{i} \otimes 1 \\
& =q_{i j}\left(x_{j} \otimes 1\right)\left(x_{i} \otimes 1\right),
\end{aligned}
$$

and similarly $\left(1 \otimes x_{i}\right)\left(1 \otimes x_{j}\right)=q_{i j}\left(1 \otimes x_{j}\right)\left(1 \otimes x_{i}\right)$ whenever $i$ and $j$ are both in $\left\{c_{1}+1, \ldots, c\right\}$. If $i \in I$ and $j \in\left\{c_{1}+1, \ldots, c\right\}$, then the degree of $x_{j}$ in $A_{\mathbf{q}_{2}}^{\mathbf{a}_{c_{2}}}$ is the unit vector $e_{j}$ in $\mathbb{Z}^{c_{2}}$. In this case we obtain the equalities

$$
\left(x_{i} \otimes 1\right)\left(1 \otimes x_{j}\right)=t(0 \mid 0) x_{i} \otimes x_{j}=x_{i} \otimes x_{j}
$$

and

$$
\left(1 \otimes x_{j}\right)\left(x_{i} \otimes 1\right)=t\left(e_{i} \mid e_{j}\right) x_{i} \otimes x_{j}=q_{j i} x_{i} \otimes x_{j},
$$

and consequently $A_{\mathbf{q}}^{\mathbf{a}_{c}}$ is isomorphic to $A_{\mathbf{q}_{1}}^{\mathbf{a}_{c_{1}}} \otimes_{k}^{t} A_{\mathbf{q}_{2}}^{\mathbf{a}_{c_{2}}}$.

The final result of this section shows that the cohomology of a twisted tensor product of graded modules is the tensor product of the respective cohomologies.

Theorem 2.2. $\mathrm{BeO}$, Theorem 3.7] Let $A$ and $B$ be abelian groups, and let $\Lambda$ and $\Gamma$ be an $A$-graded and a B-graded $k$-algebra, respectively. Furthermore, let $t: A \otimes_{\mathbb{Z}} B \rightarrow k \backslash\{0\}$ be a homomorphism, and let $M_{1}, M_{2} \in \operatorname{grmod} \Lambda$ and $N_{1}, N_{2} \in$ grmod $\Gamma$ be graded modules. Then there is an isomorphism

$$
\operatorname{Ext}_{\Lambda \otimes_{k}^{t} \Gamma}^{*}\left(M_{1} \otimes_{k}^{t} N_{1}, M_{2} \otimes_{k}^{t} N_{2}\right) \simeq \operatorname{Ext}_{\Lambda}^{*}\left(M_{1}, M_{2}\right) \otimes_{k} \operatorname{Ext}_{\Gamma}^{*}\left(N_{1}, N_{2}\right)
$$

of graded vector spaces.

\section{Ext-SyMmetry}

In this section, we prove that symmetry holds in the vanishing of cohomology for graded modules over a quantum complete intersection, provided the commutators are all roots of unity. The idea of the proof is to use twisted tensor products to pass to a bigger quantum complete intersection where Ext-symmetry holds, namely a symmetric one. In order to do this, we must determine precisely when a quantum complete intersection is symmetric. Throughout this section, we fix a field $k$.

Recall that a finite dimensional $k$-algebra $\Lambda$ is Frobenius if $\Lambda \Lambda$ and $D\left(\Lambda_{\Lambda}\right)$ are isomorphic as left $\Lambda$-modules, where $D$ denotes the usual $k$-dual $\operatorname{Hom}_{k}(-, k)$. If $\Lambda$ and $D(\Lambda)$ are isomorphic as bimodules, then $\Lambda$ is symmetric. Now suppose $\Lambda$ is Frobenius, and let $\phi:{ }_{\Lambda} \Lambda \rightarrow D\left(\Lambda_{\Lambda}\right)$ be an isomorphism. Let $y \in \Lambda$ be any element, and consider the linear functional $\phi(1) \cdot y \in D(\Lambda)$, i.e. the $k$-linear map $\Lambda \rightarrow k$ defined by $\lambda \mapsto \phi(1)(y \lambda)$. Since $\phi$ is surjective, there is an element $x \in \Lambda$ having the property that $\phi(x)=\phi(1) \cdot y$, giving $x \cdot \phi(1)=\phi(1) \cdot y$ since $\phi$ is a map of left $\Lambda$-modules. It is not difficult to show that the map $y \mapsto x$ defines a $k$-algebra automorphism on $\Lambda$, and its inverse $\nu$ is called the Nakayama automorphism of $\Lambda$ (with respect to $\phi$ ). Thus $\nu$ is defined by $\phi(1)(\lambda x)=\phi(1)(\nu(x) \lambda)$ for all $\lambda \in \Lambda$. This automorphism is unique up to an inner automorphism; if $\phi^{\prime}:{ }_{\Lambda} \Lambda \rightarrow D\left(\Lambda_{\Lambda}\right)$ is another isomorphism of left modules yielding a Nakayama automorphism $\nu^{\prime}$, then there exists an invertible element $z \in \Lambda$ such that $\nu=z \nu^{\prime} z^{-1}$. Note that $\phi$ is an isomorphism between the bimodules ${ }_{1} \Lambda_{\nu^{-1}}$ and $D(\Lambda)$. Moreover, note that $\Lambda$ is symmetric if and only if the Nakayama automorphism is the identity.

As $D\left(\Lambda_{\Lambda}\right)$ is an injective left $\Lambda$-module, we see that a Frobenuis algebra is always left selfinjective, but in fact the definition is left-right symmetric. For if 
$\phi:{ }_{\Lambda} \Lambda \rightarrow D\left(\Lambda_{\Lambda}\right)$ is an isomorphism of left $\Lambda$-modules, we can dualize and get an isomorphism $D(\phi): D^{2}\left(\Lambda_{\Lambda}\right) \rightarrow D\left({ }_{\Lambda} \Lambda\right)$ of right modules. Composing with the natural isomorphism $\Lambda_{\Lambda} \simeq D^{2}\left(\Lambda_{\Lambda}\right)$, we obtain an isomorphism $\Lambda_{\Lambda} \rightarrow D\left({ }_{\Lambda} \Lambda\right)$ of right $\Lambda$-modules.

A finite dimensional local algebra is Frobenius if and only if it is selfinjective. In particular, a quantum complete intersection $A_{\mathbf{q}}^{\mathbf{a}_{c}}$ is Frobenius, and the following result shows that there is a particularly nice Nakayama automorphism.

Lemma 3.1. A quantum complete intersection $A_{\mathbf{q}}^{\mathbf{a}_{c}}$ is Frobenius, with a Nakayama automorphism $A_{\mathbf{q}}^{\mathbf{a}_{c}} \stackrel{\nu}{\rightarrow} A_{\mathbf{q}}^{\mathbf{a}_{c}}$ given by

$$
x_{w} \mapsto\left(\prod_{i=1}^{c} q_{i w}^{a_{i}-1}\right) x_{w}
$$

for $1 \leq w \leq c$

Proof. Consider the map $A_{\mathbf{q}}^{\mathbf{a}_{c}} \stackrel{\phi}{\rightarrow} D\left(A_{\mathbf{q}}^{\mathbf{a}_{c}}\right)$ defined by

$$
\phi(1): \sum_{i_{1}, \ldots, i_{c}} \alpha_{i_{1}, \ldots, i_{c}} x_{c}^{i_{c}} \cdots x_{1}^{i_{1}} \mapsto \alpha_{a_{1}-1, \ldots, a_{c}-1}
$$

That is, the element $\phi(1)$ maps an element $y \in A_{\mathbf{q}}^{\mathbf{a}_{c}}$ to the coefficient of the socle element $x_{c}^{a_{c}-1} \cdots x_{1}^{a_{1}-1}$ in $y$. This is an isomorphism of left $A_{\mathbf{q}}^{\mathbf{a}_{c}}$-modules. By definition, a Nakayama automorphism $A_{\mathbf{q}}^{\mathbf{a}_{c}} \stackrel{\nu}{\rightarrow} A_{\mathbf{q}}^{\mathbf{a}_{c}}$ has the property that $y \cdot \phi(1)=$ $\phi(1) \cdot \nu(y)$ for all $y \in A_{\mathbf{q}}^{\mathbf{a}_{c}}$. The given map satisfies this property.

Thus quantum complete intersections are not symmetric in general. However, the following result shows that for every such algebra, there exists a symmetric quantum complete intersection "extending" the given one.

Proposition 3.2. Given any quantum complete intersection $A_{\mathbf{q}}^{\mathbf{a}_{c}}$, there exists a symmetric quantum complete intersection $A_{\mathbf{q}^{\prime}}^{\mathbf{a}_{2 c}}$ with the following properties:

(i) The subalgebra of $A_{\mathbf{q}^{\prime}}^{\mathbf{a}_{2 c}}$ generated by $x_{1}, \ldots, x_{c}$ is isomorphic to $A_{\mathbf{q}}^{\mathbf{a}_{c}}$.

(ii) The commutators of $A_{\mathbf{q}^{\prime}}^{\mathbf{a}_{2 c}}$ are the commutators of $A_{\mathbf{q}}^{\mathbf{a}_{c}}$.

Proof. Suppose $A_{\mathbf{q}}^{\mathbf{a}_{c}}$ is given by the sequence $\mathbf{a}_{c}=\left(a_{1}, \ldots, a_{c}\right)$ and the commutation matrix

$$
\mathbf{q}=\left(\begin{array}{ccc}
q_{11} & \cdots & q_{1 c} \\
\vdots & \ddots & \vdots \\
q_{c 1} & \cdots & q_{c c}
\end{array}\right)
$$

in which $q_{i j} q_{j i}=1$ and $q_{i i}=1$ for all $i, j$. Define a sequence $\mathbf{a}_{2 c}$ and a $2 c \times 2 c$ commutation matrix $\mathbf{q}^{\prime}$ by

$$
\begin{aligned}
\mathbf{a}_{2 c} & \stackrel{\text { def }}{=}\left(a_{1}, \ldots, a_{c}, a_{1}, \ldots, a_{c}\right) \\
\mathbf{q}^{\prime} & \stackrel{\text { def }}{=}\left(\begin{array}{cc}
\mathbf{q} & \mathbf{q}^{\mathrm{T}} \\
\mathbf{q}^{\mathrm{T}} & \mathbf{q}
\end{array}\right)
\end{aligned}
$$

where $Q^{\mathrm{T}}$ denotes the transpose of a matrix. Then $A_{\mathbf{q}}^{\mathbf{a}_{c}}$ is isomorphic to the subalgebra of the quantum complete intersection $A_{\mathbf{q}^{\prime}}^{\mathbf{a}_{2 c}}$ generated by $x_{1}, \ldots, x_{c}$. The commutators $q_{u v}^{\prime}$ in $A_{\mathbf{q}^{\prime}}^{\mathbf{a}_{2 c}}$ satisfy

$$
q_{u v}^{\prime}= \begin{cases}q_{u v} & \text { if } u \leq c, v \leq c \\ q_{v i} & \text { if } u=c+i, v \leq c \\ q_{j u} & \text { if } u \leq c, v=c+j \\ q_{i j} & \text { if } u=c+i, v=c+j\end{cases}
$$


and so if $i$ and $w$ are integers with $1 \leq i \leq c$ and $1 \leq w \leq 2 c$, we see that $q_{i w}^{\prime} q_{(c+i) w}^{\prime}=1$. This gives

$$
\prod_{i=1}^{2 c}\left(q_{i w}^{\prime}\right)^{a_{i}-1}=\left(\prod_{i=1}^{c}\left(q_{i w}^{\prime}\right)^{a_{i}-1}\right)\left(\prod_{i=1}^{c}\left(q_{(c+i) w}^{\prime}\right)^{a_{i}-1}\right)=1
$$

for $1 \leq w \leq 2 c$, and therefore $A_{\mathbf{q}^{\prime}}^{\mathbf{a}_{2 c}}$ is symmetric by Lemma 3.1.

As mentioned, the crucial step when proving Ext-symmetry for graded modules over a quantum complete intersection is the passing to a bigger symmetric algebra, as in the previous result. Namely, the next result shows that Ext-symmetry holds for all modules over a symmetric quantum complete intersection whose commutators are all roots of unity. Recall first the following; details can be found in $\underline{\mathrm{SnS}}$ and $\underline{\mathrm{Sol}}$. Let $\Lambda$ a finite dimensional $k$-algebra, and denote the enveloping algebra $\Lambda \otimes_{k} \Lambda^{\text {op }}$ of $\Lambda$ by $\Lambda^{\mathrm{e}}$. For $n \geq 0$, the $n$th Hochschild cohomology group of $\Lambda$, denoted $\operatorname{HH}^{n}(\Lambda)$, is the vector space $\operatorname{Ext}_{\Lambda^{\mathrm{e}}}^{n}(\Lambda, \Lambda)$. The graded vector space $\operatorname{HH}^{*}(\Lambda)=\operatorname{Ext}_{\Lambda^{\mathrm{e}}}^{*}(\Lambda, \Lambda)$ is a graded-commutative ring with Yoneda product, and for every $M \in \bmod \Lambda$ the tensor product $-\otimes_{\Lambda} M$ induces a homomorphism

$$
\mathrm{HH}^{*}(\Lambda) \stackrel{\varphi_{M}}{\longrightarrow} \operatorname{Ext}_{\Lambda}^{*}(M, M)
$$

of graded $k$-algebras. If $N \in \bmod \Lambda$ is another module and $\eta \in \mathrm{HH}^{*}(\Lambda)$ and $\theta \in$ $\operatorname{Ext}_{\Lambda}^{*}(M, N)$ are homogeneous elements, then the relation $\varphi_{N}(\eta) \circ \theta=(-1)^{|\eta||\theta|} \theta \circ$ $\varphi_{M}(\eta)$ holds, where "o" denotes the Yoneda product. In the terminology used in Ber] and BIKO, the Hochschild cohomology ring $\operatorname{HH}^{*}(\Lambda)$ acts centrally on the bounded derived category $D^{b}(\Lambda)$ of $\bmod \Lambda$.

Theorem 3.3. Let $A_{\mathbf{q}}^{\mathbf{a}_{c}}$ be a symmetric quantum complete intersection whose commutators $q_{i j}$ are all roots of unity. Then for all modules $M, N \in \bmod A_{\mathbf{q}}^{\mathbf{a}_{c}}$, the following are equivalent:

(i) $\operatorname{Ext}_{A_{\mathbf{q}}^{\mathbf{a}_{\mathbf{c}}}}^{i}(M, N)=0$ for $i \gg 0$.

(ii) $\operatorname{Ext}_{A_{\mathrm{q}}^{\mathrm{a}}}^{i}(M, N)=0$ for $i>0$.

(iii) $\operatorname{Ext}_{A_{\mathbf{q}}^{\mathbf{a}_{c}}}^{i}(N, M)=0$ for $i \gg 0$.

(iv) $\operatorname{Ext}_{A_{\mathbf{q}}^{\mathbf{a}_{c}}}^{i}(N, M)=0$ for $i>0$.

Proof. By [BeO, Theorem 5.5], the Hochschild cohomology ring $\mathrm{HH}^{*}\left(A_{\mathbf{q}}^{\mathbf{a}_{c}}\right)$ is Noetherian, and $\operatorname{Ext}_{A_{\mathbf{q}}^{\mathbf{a}_{c}}}^{i}(k, k)$ is a finitely generated $\operatorname{HH}^{*}\left(A_{\mathbf{q}}^{\mathbf{a}_{c}}\right)$-module. The result now follows from [Ber, Theorem 4.2].

Examples. (i) Let $A$ be the exterior algebra on a $c$-dimensional $k$-vector space, i.e.

$$
A=k\left\langle X_{1}, \ldots, X_{c}\right\rangle /\left(X_{i}^{2},\left\{X_{i} X_{j}+X_{j} X_{i}\right\}_{i \neq j}\right) .
$$

From Lemma 3.1 we see that when applying the Nakayama automorphism to a generator $x_{i}$, then the result is the element $(-1)^{c-1} x_{i}$. Therefore $A$ is symmetric precisely when $c$ is an odd number. Consequently, symmetry in the vanishing of cohomology holds for modules over exterior algebras on odd-dimensional vector spaces (cf. [Mor, Corollary 4.9]).

(ii) Fix integers $c$ and $a$, both at least two, and let $q$ be an element in $k$ with the property that $q^{a-1}=1$. Furthermore, let $\mathbf{a}_{c}$ be the $c$-tuple $(a, \ldots, a)$, let $\mathbf{q}$ be the commutation matrix

$$
\left(\begin{array}{ccccc}
1 & q & \cdots & \cdots & q \\
q^{-1} & 1 & q & \cdots & q \\
\vdots & \ddots & \ddots & \ddots & \vdots \\
q^{-1} & \cdots & q^{-1} & 1 & q \\
q^{-1} & \cdots & \cdots & q^{-1} & 1
\end{array}\right)
$$


and consider the quantum complete intersection $A_{\mathbf{q}}^{\mathbf{a}_{c}}$. Explicitly, this is the algebra

$$
k\left\langle X_{1}, \ldots, X_{c}\right\rangle /\left(X_{i}^{a},\left\{X_{i} X_{j}-q X_{j} X_{i}\right\}_{i<j}\right),
$$

and from Lemma 3.1 we see that it is symmetric. Thus Ext-symmetry holds for all modules over $A_{\mathbf{q}}^{\mathbf{a}_{c}}$.

Using Theorem 3.3 we now show that Ext-symmetry holds for all graded modules over an arbitrary quantum complete intersection whose commutators are all roots of unity.

Theorem 3.4. Let $A_{\mathbf{q}}^{\mathbf{a}_{c}}$ be a quantum complete intersection whose commutators $q_{i j}$ are all roots of unity. Then for all modules $M, N \in \operatorname{grmod} A_{\mathbf{q}}^{\mathbf{a}_{c}}$, the following are equivalent:

(i) $\operatorname{Ext}_{A_{\mathbf{q}}^{\mathbf{a}_{c}}}^{i}(M, N)=0$ for $i \gg 0$.

(ii) $\operatorname{Ext}_{A_{\mathbf{q}}^{\mathbf{a}_{c}}}^{i}(M, N)=0$ for $i>0$.

(iii) $\operatorname{Ext}_{A_{\mathbf{q}}^{\mathbf{a}_{c}}}^{i}(N, M)=0$ for $i \gg 0$.

(iv) $\operatorname{Ext}_{A_{\mathbf{q}}^{\mathbf{a}_{c}}}^{i}(N, M)=0$ for $i>0$.

Proof. Let $A_{\mathbf{q}^{\prime}}^{\mathbf{a}_{2 c}}$ be a symmetric quantum complete intersection with the properties given in Proposition 3.2. That is, the subalgebra of $A_{\mathbf{q}^{\prime}}^{\mathbf{a}_{2 c}}$ generated by $x_{1}, \ldots, x_{c}$ is isomorphic to $A_{\mathbf{q}}^{\mathbf{a}_{c}}$, and the commutators of $A_{\mathbf{q}^{\prime}}^{\mathbf{a}_{2 c}}$ are the commutators of $A_{\mathbf{q}}^{\mathbf{a}_{c}}$. By Lemma 2.1, there exists a homomorphism $\mathbb{Z}^{c} \otimes_{\mathbb{Z}} \mathbb{Z}^{c} \stackrel{t}{\rightarrow} k \backslash\{0\}$ and a quantum complete intersection $A_{\mathbf{p}}^{\mathbf{b}_{c}}$, such that $A_{\mathbf{q}^{\prime}}^{\mathbf{a}_{2 c}}$ is isomorphic to the twisted tensor product $A_{\mathbf{q}}^{\mathbf{a}_{c}} \otimes_{k}^{t} A_{\mathbf{p}}^{\mathbf{b}_{c}}$. Now for graded $A_{\mathbf{q}}^{\mathbf{a}_{c}}$-modules $M$ and $N$, Theorem 2.2 provides an isomorphism

$$
\operatorname{Ext}_{A_{\mathbf{q}^{\prime}}^{\mathbf{a}_{2}}}^{*}\left(M \otimes_{k}^{t} A_{\mathbf{p}}^{\mathbf{b}_{c}}, N \otimes_{k}^{t} A_{\mathbf{p}}^{\mathbf{b}_{c}}\right) \simeq \operatorname{Ext}_{A_{\mathbf{q}}^{\mathbf{a}_{c}}}^{*}(M, N) \otimes_{k} \operatorname{Ext}_{A_{\mathbf{p}}^{\mathbf{b}_{c}}}^{*}\left(A_{\mathbf{p}}^{\mathbf{b}_{c}}, A_{\mathbf{p}}^{\mathbf{b}_{c}}\right)
$$

of graded vector spaces. The result now follows from Theorem 3.3, since all the commutators of $A_{\mathbf{q}^{\prime}}^{\mathbf{a}_{2 c}}$ are roots of unity.

\section{REFERENCES}

[AvB] L. Avramov, R.-O. Buchweitz, Support varieties and cohomology over complete intersection, Invent. Math. 142 (2000), 285-318.

[Ber] P. A. Bergh, On the vanishing of cohomology in triangulated categories, preprint.

[BIKO] P. A. Bergh, S. Iyengar, H. Krause, S. Oppermann, Dimensions of triangulated categories via Koszul objects, preprint.

[BeO] P. A. Bergh, S. Oppermann, Cohomology of twisted tensor products, J. Algebra 320 (2008), no. 8, 3327-3338.

[Mor] I. Mori, Symmetry in the vanishing of Ext over stably symmetric algebras, J. Algebra 310 (2007), no. 2, 708-729.

[SnS] N. Snashall, Ø. Solberg, Support varieties and Hochschild cohomology rings, Proc. London Math. Soc. (3) 88 (2004), no. 3, 705-732.

[Sol] $\varnothing$. Solberg, Support varieties for modules and complexes, in Trends in representation theory of algebras and related topics, 239-270, Contemp. Math., 406, Amer. Math. Soc., Providence, RI, 2006.

Petter Andreas Bergh, Institutt for matematiske fag, NTNu, N-7491 Trondheim, NORWAY

E-mail address: bergh@math.ntnu.no 